\title{
Enzyme catalysed tandem reactions
}

Isabel Oroz-Guinea ${ }^{1}$ and Eduardo García-Junceda ${ }^{1}$

${ }^{1}$ Departamento de Química Bio-Orgánica, Instituto de Química Orgánica General, CSIC. Juan de la Cierva 3. 28006 Madrid, Spain.

Corresponding author: Isabel Oroz-Guinea (isabel.oroz@iqog.csic.es) and Eduardo GarcíaJunceda (eduardo.junceda@csic.es)

\begin{abstract}
To transfer to the laboratory, the excellent efficiency shown by enzymes in Nature, biocatalysis, had to mimic several synthetic strategies used by living organisms. Biosynthetic pathways are examples of tandem catalysis and may be assimilated in the biocatalysis field for the use of isolated multienzyme systems in the homogeneous phase. The concurrent action of several enzymes that work sequentially presents extraordinary advantages from the synthetic point of view since it permits to become irreversible a reversible process, to shift the equilibrium reaction in such a way that enantiopure compounds can be obtained from prochiral or racemic substrates, reduce or eliminate problems due to product inhibition or prevent the shortage of substrates by dilution or degradation in the bulk media, etc. In this review we want to illustrate the developments of recent studies involving in vitro multi-enzyme reactions for the synthesis of different classes of organic compounds.
\end{abstract}

\section{Introduction}

Chemists have always been inspired by Nature, not only due to the vast diversity of compounds that living organisms are capable of creating, but also due to the extraordinary synthetic strategies deployed. Inside of a cell, thousands of different chemical reactions take place to support cellular growth and survival. Enzymes, the catalysts used by living organisms, have evolved to perform these diverse chemical transformations with high selectivity and specificity. This extraordinary synthetic effectiveness that enzymes display is due in part to their work in multi-step reactions, or cascade processes which give rise to the complex metabolic networks found in biological systems. This synthetic strategy allows building complex structures from simple elements, can make irreversible a reversible process, eliminates inhibition problems caused by product excess, or prevents the lack of substrate $\left[1^{\bullet \bullet}\right]$.

Biocatalysts have adopted Nature's strategy to reproduce such tandem reactions and to apply them in organic synthesis as a powerful tool to efficiently produce structurally complex molecules. Two major approaches have been made: the use of whole microorganisms (in vivo) 
or the use of isolated enzymes (in vitro) $\left[2^{\bullet \bullet}\right]$. In vivo approaches exploit the metabolic pathways of the microbial hosts, which often have been engineered to produce the desired product, and to minimize the diversion of substrate for their normal metabolic requirements. The use of microorganisms has some advantages: i) there is no need for enzyme isolation or purification; ii) enzymes show a high stability due to the fact that they are surrounding of their natural environment and iii) cofactor regeneration is typically provided by the cell own metabolic machinery. Although the complexity of the cellular system provides all these benefits, has also some drawbacks: i) engineering microorganisms is labor-intensive and time consuming; ii) lower selectivities are achieved owing to the competition from other similar enzymes present in the cell; iii) a balance between the toxicity of the substance produced and the product yield is required and iv) control and maximization of the synthetic processes is difficult. In contrast, in vitro approaches are less complex and, therefore, reaction conditions are easier to control and can be optimized by, for example, varying the concentration of the enzyme or the substrate, adding co-solvents or with variation of $\mathrm{pH}$ or temperature. Also a higher purity product is usually obtained due to the absence of competing side reactions and metabolites which are present in in vivo systems. Despite these advantages there are some limitations: i) isolation, characterization and obtaining sufficient quantities of the enzymes for the reaction are required; ii) the enzymes have to be stable and functional under reaction conditions; iii) cofactor dependent enzyme reactions need a continuous cofactor regeneration system to avoid the use of stoichiometric amount of usually expensive co-factors. Alternatives to overcome these limitations included the use of recombinant DNA techniques to identify, produce and modified proteins; enzyme immobilization that can enhance their stability and allow the possibility to compartmentalize synthetic routes incompatible among them and the use of enzymatic cofactor regeneration systems.

In this review we want to illustrate the developments of recent studies involving in vitro multienzyme reactions for the synthesis of different compounds.

\section{Tandem reactions for the preparation of alcohols}

Alcohols are useful intermediates or building blocks for the synthesis of many pharmaceuticals and chemicals. These compounds are produce in Nature following several pathways and using different classes of enzymes like oxidoreductases, hydrolases and lyases.

In general, the use of oxidoreductases (OR) allows the transfer of electrons from a reductant to an oxidant, catalyzing a redox process and producing alcohol through the reduction of a carbonyl compounds. Ketoreductases (KREDs), provide alcohols via ketone reduction. BaeyerVilliger monooxygenases (BVMOs) are another type of redox enzymes which catalyze the 
oxidation of ketones, sulfides, and other heteroatoms by atmospheric oxygen. All these enzymes are ubiquitous in Nature and the number of new identified members of these families is continually increasing. The use of OR presents a main limitation since most of them are dependent on the nicotinamide cofactors $\beta$-1,4-nicotinamide adenindinucleotide (NADH), $\beta$ 1,4-nicotinamide adenine dinucleotide phosphate (NADPH) or rarely cofactors from the group of the flavines (FAD) or methoxatines. Thus, cofactor regeneration is needed attending to the expensive cost of these compounds. Effective cofactor regeneration methods as chemical, electrochemical, photochemical or enzymatic methods have been developed being the multienzymatic systems for in situ co-factor regeneration approach the methodology most often exploited. Excellent literature revisions about this subject have been published $[3,4]$ thus, in this paper enzymatic strategies for cofactor regeneration will be not discussed.

There are several examples for the use of KREDS in literature. Wada et al. [5] obtained the enantiopure alcohol (4R, 6R)-actinol (4-hydroxy-2,2,6-trimethylcyclohexanone) from ketoisophorone [3,5,5-trimethyl-2- cyclohexene-1,4-dione] by a two-step enzymatic asymmetric reduction system which started with the asymmetric reduction of the $\mathrm{C}=\mathrm{C}$ bond followed by the asymmetric reduction of (6R)-levodione to $(4 R, 6 R)$-actinol using the old yellow enzyme from Saccharomyces cerevisiae and the (6R)-levodione reductase of Corynebacterium aquaticum M13. Glucose dehydrogenase was also used for cofactor regeneration in both steps (Figure 1A). Ma et al. [6] synthesized the ethyl (R)-4-cyano-3-hydroxybutyrate, a key intermediate in the synthesis of the cholesterol-lowering drug atorvastatin (Lipitor). This compound can be prepared by KRED catalysed reduction of a $\beta$-keto ester coupled with a GDH catalysed conversion of glucose to gluconate for cofactor regeneration. A third enzyme, halohydrin dehalogenase $(\mathrm{HHDH})$ is employed for the subsequent conversion of the chlorohydrin product to the corresponding cyano compound (Figure 1B). Also enantiomerically pure diols can be achieved combining the use of two different ketoreductases by two consecutive reduction reactions: a stereoselective diketone reduction and a hydroxy ketone reduction [7].

One-pot deracemization of secondary alcohols can be performed by sequential ADHs oxidation and reduction processes. An artificial reaction pathway combining simultaneous concurrent tandem oxidation and reduction cycles with opposite cofactor and stereopreference in one-pot was designed by Voss et al. [8 ${ }^{\bullet \bullet}$ ] for the resolution of alcohol racemates. Both enantipure $(R)$ or $(S)$-alcohols were obtained combining different estereoseltive ADHs with an oxidase and a formate dehydrogenase for the cofactor regeneration. This approach can be extended to stereoinversion of enantioenriched sec-alcohols (Figure 1C). Another way for the deracemisation of secondary alcohols is the enantioselective oxidation of several sec-alcohols by an ADH combined with the stereoselective oxidation of different ketones or sulfides by a BVMO. Both reactions are productive and connected through internal cofactor recycling, 
A

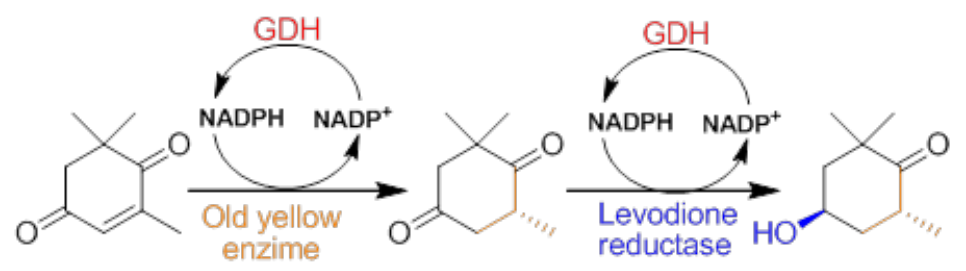

$\mathrm{B}$
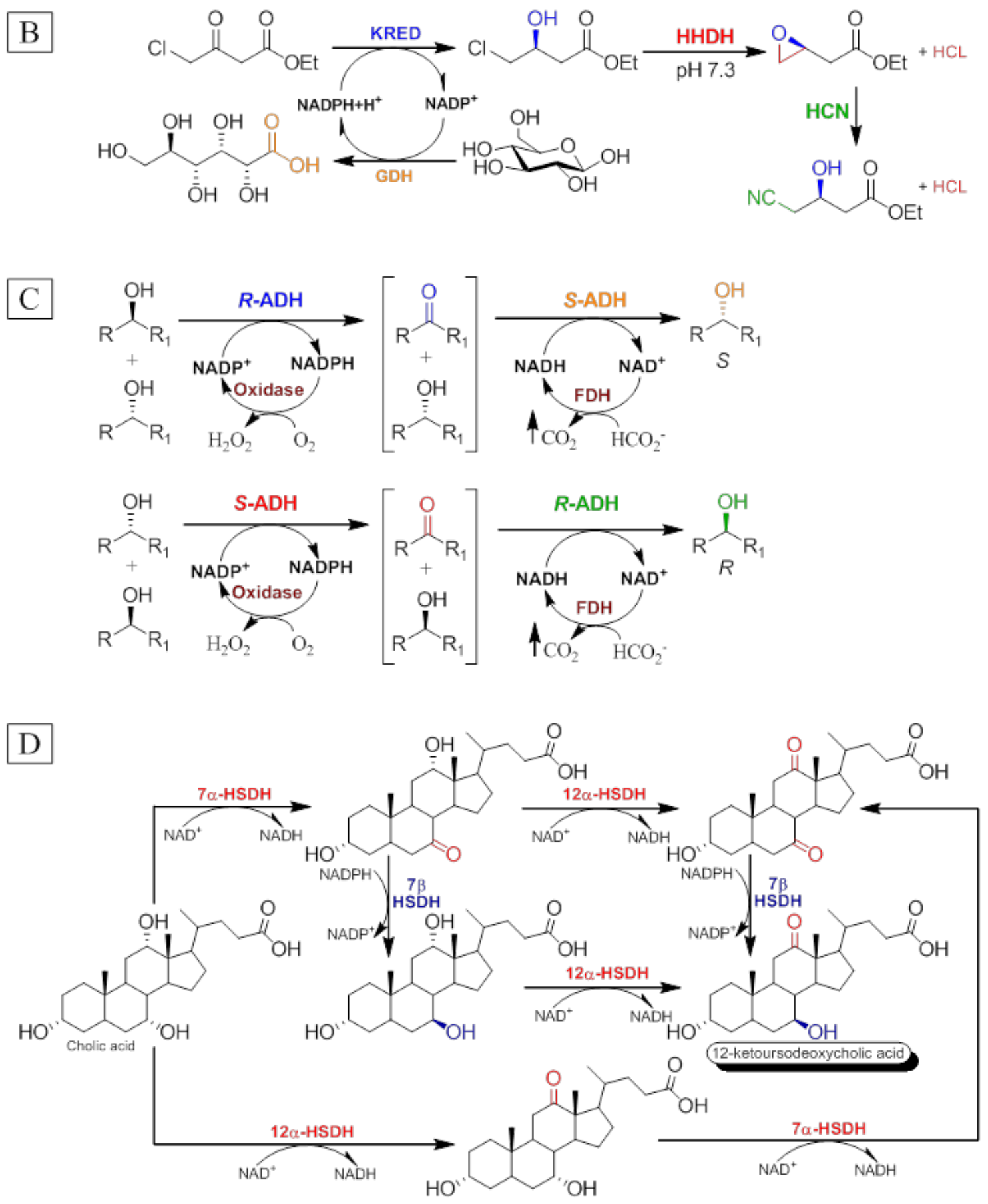

Figure 1. Multi-enzyme syntheses of alcohols. A. Synthesis of $(4 R, 6 R)$-actinol from ketoisophorone. B. Synthesis of ethyl (R)-4-cyano-3-hydroxybutyrate. C. Sequential oxidation and reduction for deracemization of racemic alcohols. D. Synthesis of 12-ketoursodeoxycholic acid. Recycling of cofactors has been omitted for a major clarity of the scheme. 
allowing a parallel interconnected kinetic asymmetric transformation (PIKAT) and maximizing the redox efficiency. In this manner, it was possible to obtain simoultaneously up to three enantioenriched derivatives $\left[9,10^{\circ}\right]$. Other example of the utilization of these enzymes is the synthesis of 12-ketoursodeoxycholic acid that was achieved by alternating oxidative and reductive steps catalysed by hydroxysteroid dehydrogenases (HSDHs) with different cofactor specificity, using the regeneration systems as the driving force. The enzymes used in this synthesis were the NADH-dependent $12 \alpha-\mathrm{HSDH}$ and $7 \alpha-\mathrm{HSDH}$ in the oxidative step and a NADPH-dependent 7 $\beta$-HSDH in the reductive one. Lactate dehydrogenase (LDH) from rabbit muscle and pyruvate were used for $\mathrm{NAD}^{+}$regeneration and NADPH was recycled by the glucose/glucose dehydrogenase system from Thermoplasma acidophilum [11] (Figure 1D). An example of a multi-step oxidative cascade involving a cytochrome P450 monooxygenase (TamI) and an FAD-dependent oxidase (TamL) have described by Carlson et al [12]. These enzymes act co-dependently through repeated exchange of substrates for post-assembly line tailoring of tirandamycin antibiotics.

Lyases and hydrolases can also be employed for the synthesis of alcohols. Lyases catalyze the

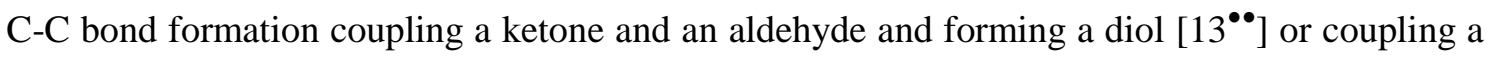
ketone with hydrogen cyanide giving an acid derivative [14], some examples will be explained subsequently. Hydrolases catalyze the hydrolytic cleavage of the carbon-oxygen single bonds by lipases or esterases in esters or analogous carboxylic derivatives to produce acids and alcohols (or rarely starting from sulphate or phosphate esters as a substrate for phosphatases or sulphatases, respectively) or by epoxide hydrolases which catalyze the opening of epoxides [15].

\section{Multi-enzyme synthesis of acids and acid derivatives}

Acids and their derivatives are very attractive for organic synthesis because of the easy transformation of the functional groups in these molecules. Two major approaches have been described in literature to prepare acids and derivatives: i) oxidation of an alcohol group and ii) hydrolysis of a cyano group catalysed by nitrilases or nitrile hydratases giving rise consecutively to carboxilic acids or amides. When amides are obtained they can be hydrolyzed to the corresponding acids by amidases. An example of the first has been reported by Di Genaro et al. [16]. These authors use sequentially an aldoketo reductase from E. coli JM109 (ECAKR), an alcohol dehydrogenase from Lactobacillus brevis (LBADH) and a dihydrodiol dehydrogenase from Pseudomonas fluorescens N3 (NDDH). The second procedure was used for the production of the $\alpha$-hidroxyacid (S)-mandelic acid. Its synthesis began with the production of (S)-mandelonitrile catalysed by a cross-linked enzyme aggregate (CLEA) of hydroxynitrile lyase from Manihot esculenta coupled with its in situ hydrolysis catalysed by a 
selective nitrilase from Pseudomonas flurescens [14]. The addition of a third enzyme, penicillin amidase, which catalyzes the hydrolysis of the (S)-mandelamide increases (S)-mandelic acid yield from $40 \%$ up to $96 \%$ with an enantiomeric excess up to $99 \%$ [4]. In the same way, $\beta$ hydroxy carboxylic acids can be obtained by the reduction of $\beta$-ketonitriles and the subsequently nitrilase-catalysed hydrolysis of the obtained optically $\beta$-hydroxy nitriles. Ankati et al. $\left[17^{\circ}\right]$ reported this reaction to afford optically active $\beta$-hydroxy carboxylic acids using a combination of a reductase from Candida magnoliae (CMCR) and a nitrilase from Cyanobacterium synechocystis sp. strain PCC 6803 (NIT6803) to obtain the (R)-enantiomer or a an alcohol dehydrogenase (Ymr226c) from Saccharomyces cerevisiae and a nitrilase from Bradyrhizobium japonicum strain USDA110 (bll6402) for the preparation of the (S)-enantiomer. By analogy with the synthesis of $\alpha$-hydroxy acids, a one-pot synthesis of $\alpha$-hydroxy amides from aldehydes via hydrocyanation and in situ nitrile hydratase-catalysed hydrolysis to the amide can be envisioned. Recently Sheldon's group [18] has design a bienzymatic cascade reaction catalysed by hidroxynitrile lyase $(\mathrm{HnL})$ and a nitrile hydratase (NHase) for the production of $\alpha$-hydroxycarboxylic amides. In this case the low selectivity of the NHase is no longer a drawback since either an $(R)$ - or $(S)$-selective $\mathrm{HnL}$ will impart its enantioselectivity on the reaction.

\section{Multi-enzyme synthesis of amino acids and amines}

Amino acids and amines deserve a special attention as they are a highly attractive target from a biocatalyical point of view. Several multi-enzyme syntheses for the production of natural and unnatural amino acids and amines have been designed due to their demand for pharmaceutical industry to treat a range of different therapeutic conditions. In the same way that alcohol synthesis, the production of these compounds can be achieved through reductive amination or via deracemisation from a starting racemic mixture. A significant number of examples of these multi-enzyme biocatalytic processes have been compiled in several excellent reviews [19, 20]. In the present review we have chosen just a few recent examples.

Three main in vitro multi-enzyme system strategies are known for amino acid synthesis:

\subsection{Multi-enzymatic synthesis of amino acids employing amino acid dehydrogenases (AADH).}

Amino acid dehydrogenases (AADHs) are $\mathrm{NAD}(\mathrm{P})^{+}$-dependent enzymes, which convert amino acids to the corresponding keto acids and ammonia. Luckily, they are also able to catalyze the reverse reaction leading to the asymmetric synthesis of amino acids via reductive amination. Enzymatic cofactor regeneration systems have been coupled to AADH to make economically viable the process. 
In a recent work published by Resch et al., [21 ${ }^{\circ}$ ] a three-enzyme cascade reaction for the deracemisation of D,L-mandelic acid to yield enantiopure L-phenylglycine ( $>97 \%$ ee.) is described in a novel type of "dynamic kinetic asymmetric transformation” (DYKAT) system. This multi-enzyme system employs mandelate racemase in combination with D-mandelate dehydrogenase (D-MDH) and different AADHs, being the D-MDH not only part of the NADH regeneration system but also providing phenyl glyoxylate, substrate of L-AADH, from Dmandelic acid (Figure 2A) Nakajima et al. [22] described a cascade system for deracemization of D,L-alanine and D,L-leucine to the corresponding L-enantiomers with an enantiomeric excess of 99\%. Deracemization can be afforded by combining D-amino acid oxidase (D-AAO) for enantioselective oxidation and the correspondent AADH for asymmetric reductive amination. Furthermore, a formate dehydrogenase for cofactor regeneration and a catalase for hydrogen peroxide degradation in order to prevent oxidative enzyme inactivation have been added to the system. This elegant four-enzyme cascade has also been applied in the conversion of Dmethionine to L-methionine by Findrik and Vasić-Rački [23] (Figure 2B).

\subsection{Multi-enzymatic synthesis of amines and amino acids employing amino transferases} (ATs)

Amino transferases are a very useful tool for biocatalytic synthesis because their capacity for introducing amino groups into ketones or keto acids giving rise to the corresponding amines and amino acids. According to their substrate scope, they can be classified in two groups: i) $\alpha$-amino transferases, which act on the $\alpha$-amino group in amino acids and ii) $\omega$-amino transferases, which can convert amines that lack vicinal carboxylic acid functionality. Both classes require pyridoxal-5'-phosphate (PLP) as coenzyme and are able to catalyze the reverse amino group transfer, resulting in an incomplete conversion. Therefore, the development of tandem processes, which allow for shifting the equilibrium to the transfer product, are needed to overcome impediments such as serious substrate/product inhibition problems and/or equilibrium constraints.

In the lasts years, $\omega$-amino transferases have emerged as versatile and powerful biocatalysts for the obtention of a broad variety of amines. A simple method to avoid the inhibitory effect of the co-produced ketone consist on remove it using a secondary enzymatic reaction. Since alanine is usually employed as the amino donor, pyruvate removal is necessary. This can be accomplis, coupling the $\omega$-AT reaction with different enzymes which can remove in situ the pyruvate formed like lactate dehydrogenase, pyruvate decarboxylase or acetolactate synthaseA similar strategy can be used reducing the ketone product to the corresponding alcohol in a reaction catalysed by alcohol dehydrogenase. Koszelewski et al. [24] coupled a (R)-selective $\omega$-AT catalysed amine kinetic resolution with a stereoselective transamination catalysed by an $(S)$ - 
selective $\omega$-AT. Pyruvate and alanine were included in the reaction and were recycled by the addition of amino acid oxidase (D-AAO) in the first step, according to procedure previously developed by Truppo et al. [25 $5^{\circ}$ and by the use of amino acid dehydrogenase (AADH) in the second step (Figure 2C). In a recent work [26], the same group report the design of another system for amine racemisation containing just the $(R)$ - and $(S)$ - $\omega$-ATs in the presence of symmetric ketone/amine co-substrates, which served as amino-group shuttles. Combination of $\omega$-AT with an alanine amino trasferase (AlaAT) can be used for pyruvate recycling by transferring the amino group from the intermediate alanine to another keto-acid [27]. Furthermore, AspATs have been coupled to branched chain amino transferase (BCATs) for recycling the amino donor L-glutamate [28].

$\alpha$-AT have been applied in kinetic resolution of racemic substrates or in asymmetric synthesis of chiral compounds, depending if the catalysed reaction runs forward or reverse. They have been coupled with amino acid dehydrogenases (AADHs) to achieve the asymmetric synthesis of Damino acids from keto acids obtaining good yields and high enantiomeric excess values. In a first step, an amino acid dehydrogenase forms an L-amino acid which is stereoinverted by a suitable amino acid racemase to give the D-enantiomer, which serves as amino donor for the reaction catalysed by a D-amino acid aminotransferase in the transformation of a keto acid to a D-amino acid. Cofactor regeneration for the AADH is supplied by formate dehydrogenase [29] (Figure 2D). An analogous methodology has been developed, omitting the amino acid racemase, for the preparation of L-amino acids which could not be produced by direct enzymatic reductive amination as $\beta$ - and $\gamma$-branched glutamic acid derivatives [30]. Furthermore, $\alpha$-AT and AADH have also been coupled in an amino acid deracemization system by Caligiuri et al. [31]. In this process a D-amino acid oxidase from Rhodotorula gracilis and L-aspartate transaminase from $E$. coli were used. Irreversibility of the reaction was assured through the spontaneous decomposition of the $\beta$-keto sulphinic acid by-product produced by the cysteinesulphinic amino donor (Figure 2D).

\subsection{Multi-enzyme systems of ammonia lyases and aminomutases for the synthesis of amino acids .}

Multi-enzyme systems using ammonia lyases and aminomutases as biocatalysts have also been developed [32]. Enantiopure L-arylalanines (e.e.>98\%) can be prepared with good yields coupling the enzymes phenylalanine ammonia lyase (PAL) and porcine liver esterase (PLE) in a one-pot multi-enzyme system [33] (Figure 2F). Also a biocatalytic resolution of racemic $\alpha$ arylalanines to enantiopure $\beta$-arylalanines has been described using a combination of phenylalanine aminomutase (PAM) and a promiscuous alanine racemase from Pseudomonas putida [34]. Finally, Wu et al. [35] have coupled both PAM and PAL for the kinetic 
$\mathrm{A}$ $\overbrace{\mathrm{CO}_{2} \mathrm{H}}^{\mathrm{OH}} \stackrel{\begin{array}{c}\text { Mandelate } \\ \text { racemase }\end{array}}{=} \stackrel{\mathrm{Ph}}{\mathrm{OH}}_{\mathrm{CO}_{2} \mathrm{H}}^{\mathrm{O}}$
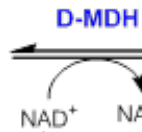

1

$\mathrm{B}$<smiles>CSCC[C@H](N)C(=O)O</smiles>

C

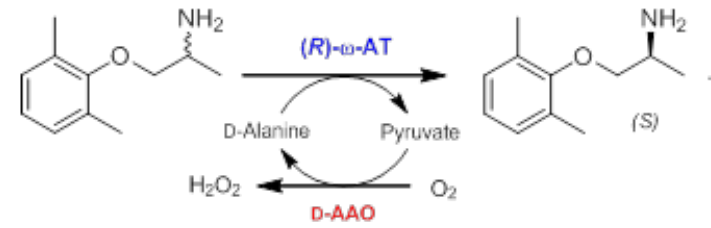<smiles>CCC(=O)COc1c(C)cccc1C</smiles><smiles>Cc1ccc(C2[C@@H]3[C@@H](C)[C@H](C(C)C)[C@@H]23)c(C)c1OCC(C)N</smiles>
DD-AADH

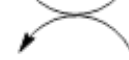

$\mathrm{NAD}^{+} \mathrm{NADH}+\mathrm{NH}^{+}$

$\mathrm{NADH}$ recyeling

$\mathrm{D}$

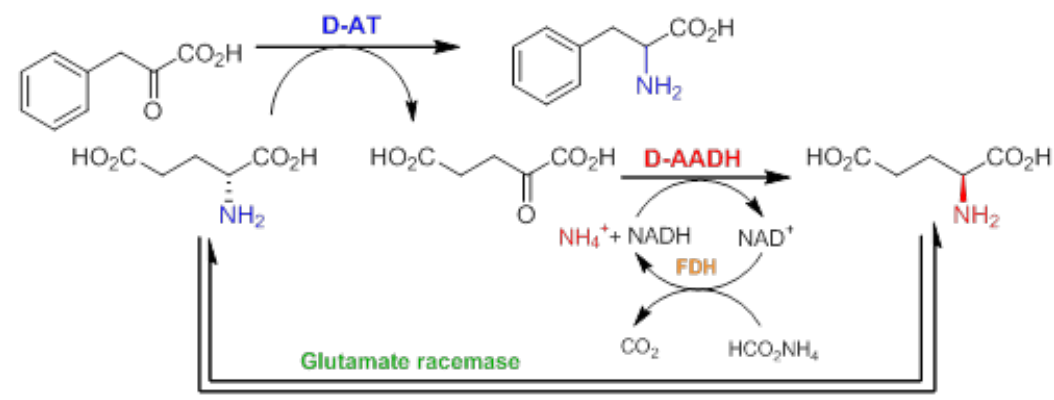

E

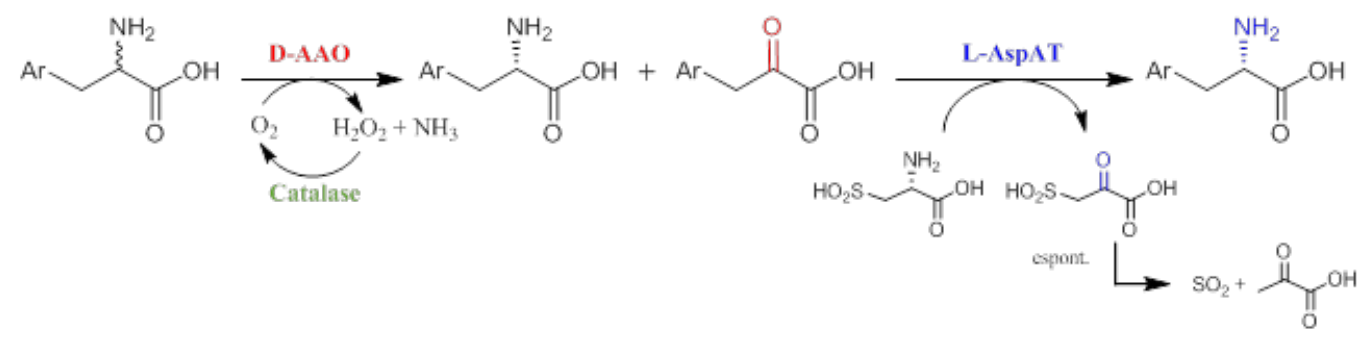

F

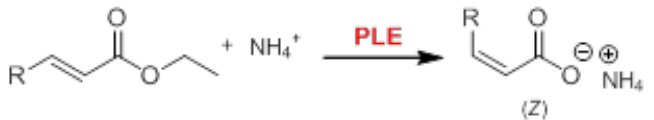

(z)<smiles>[R]C=CC(=O)O[18OH]</smiles>

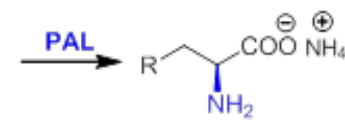

Figure 2. Tandem ezymatic reactions for the synthesis of amines and aminoacids. A. Deracemistaion of mandelic acid to yield L-phenylgycine. B. Complete conversion of D-methionine into L-methionine in a four enzymes cascade reaction.C. Deracemisation of racemic mexilene using $(R)$ - and (S)-selective $\omega$ transaminases. D. Synthesis of a D-amino acid from a $\alpha$-ketoacid using a D-selective AAO and a Lselective $\alpha$-AT. E. Deracemization of $\alpha$-amino acids. F. Synthesis of $\alpha$-arylalanines using porcine liver esterase and phenylananine ammonia lyase. 
resolution of aromatic $\beta$-amino acids. Initially, PAM catalyzes the enantioselective isomerisation of (R)- $\beta$-phenylalanines to $(S)$ - $\alpha$-phenylalanines and PAL catalyzes the deamination of the $\alpha$-amino acid formed to the corresponding cinamic acid. In the process described a wide range of $\beta$-amino acids were obtained in high enantiomeric excess (99\%).

\section{Tandem reactions for $\mathrm{C}-\mathrm{C}$ bond formation}

One of the most important reactions in organic synthesis is the formation of carbon-carbon (CC) bonds. Aldolases and hydroxynitrile lyases are the most used enzymes for C-C bond formation in biotransformations, taking part of great number of cascade processes. Previously we have described the use of hydroxynitrile lyases for acid and acid derivative synthesis, therefore, in this section we will summarize recent progress in C-C bond formation cascade reaction mediated by aldolases.

Aldolases constitute a family of lyases, which catalyze reversibly and stereoselectively an aldol addition reaction between a donor compound (nucleophile) and an acceptor compound (electrophile) leading to the formation of one or two new stereogenic centers. Usually these enzymes show high specificity towards the donor substrate but tolerate a broad range of acceptor substrates, making of them an attractive tool in the asymmetric construction of several compounds as carbohydrates, amino acids, and their analogues. According to their donor specificity aldolases can be classified into (i) pyruvate/oxaloacetate/2-oxobutyrate-dependent, (ii) dihydroxyacetone phosphate (DHAP)-dependent, (iii) dihydroxy- acetone (DHA)-dependent and other unphosphorylated analogues, (iv) threonine-dependent and (v) acetaldehydedependent aldolases $\left[13^{\bullet \bullet}\right]$.

\subsection{Tandem reactions employing pyruvate or phosphoenol pyruvate dependent aldolases.}

Pyruvate-dependent or phosphoenol pyruvate-dependent aldolases, reversibly catalyze the aldol addition of pyruvate or phosphoenol pyruvate to a variety of polyhydroxylated aldehydes. $\mathrm{N}$ Acetylneuraminic acid aldolases (NeuAc aldolases) use pyruvate as the donor substrate and catalyze the aldol reaction between pyruvate and mannose or mannose derivatives. They have been extensively studied owing their capacity to produce sialic acids derivatives used in cancer therapy and as anti-infectives. In spite of the fact that the substrate for NeuAc aldolases, $\mathrm{N}$ acetylmannosamine, is expensive and difficult to synthesize, there are a possibility to overcome this problem by in situ formation of $\mathrm{N}$-acetylmannosamine from $\mathrm{N}$-acetylglucosamine by an $\mathrm{N}$ acetylglucosamine 2-epimerase and the subsequently coupling of this reaction with the production of $\mathrm{N}$-acetylneuraminic acid by NeuAc aldolase [36]. The ability of the NeuAc aldolase to accept a broad range of substrates has been further exploited for the one-pot three enzyme synthesis of sialosides. In this system 2-mannose derivatives are obtained as acceptor 
substrates by chemical or enzymatic modification and coupled with pyruvate to form sialic acid derivatives by a sialic acid aldolase-catalysed reaction. The one-pot process included cytidine 5'-monophosphosialic acid synthetase (CMP-sialic acid synthetase) to activate the sialic acid derivative and a sialyltransferase for the formation of sialosides. It is interesting to mention that three different CMP-sialic acid synthetases were tested (Figure 3A). The use of these compounds as acceptors for the $\alpha 2-8$-sialyltransferase from Campylobacter jejuni results in GD3 ganglioside oligosaccharides and other disialyl glycans containing different natural and non-natural sialic acids [37]. In a similar approach, Cao et al. [38] used C-5-hydroxy-substituted mannose derivatives to produce a variety of C-5-substituted sialosides.

\subsection{Tandem reactions employing DHAP-dependent aldolases.}

DHAP-dependent aldolases catalyze the reversible aldol addition between DHAP and a large variety of aldehyde acceptors. The configuration of the two new stereocenters formed can be controlled by choosing one out of four stereocomplementary DHAP aldolases. One of the main drawbacks of DHAP-aldolases is their selectivity towards DHAP, which is chemically unstable and expensive. In this sense our research group has developed a straightforward multi-enzyme system for one-pot C-C bond formation catalysed by DHAP-dependent aldolases that allows the use of DHA as initial donor [39]. This system is based on the use of the recombinant ATPdependent DHAK from C. freundii CECT 4626 for the in situ phosphorylation of DHA [40]. The multi-enzyme system was completed with the regeneration of ATP catalysed by acetate kinase. This multi-enzyme system is robust enough to be used with the three synthetically useful DHAP-dependent aldolases and with aldehydes with great structural diversity [41]. The possibility to apply one-step or two-steps strategies, make the system sufficiently flexible to be able to work under different reaction conditions such as the presence of co-solvents [42 ${ }^{\bullet}$. Another method for the DHAP generation is based on the use of the acid phosphatase from Shigella flexneri (PhoN-Sf) using pyrophosphate (PPi) as phosphate donor. This enzyme firstly phosphorylates DHA and secondly, after the aldolase condensation, dephosphorylates the aldol adduct which leads to phosphate cycling while preventing the retroaldol reaction and shifting the equilibrium to completion [43] (Figure 3B).

One of the main applications of DHAP-dependent aldolases has been the synthesis of iminoand aminocyclitols, which are, in general, inhibitors of glycosidases and glycosyltransferases. A quite interesting methodology to access to nitro- and aminocyclitols has been described by Lemaire and coworkers $\left[44^{\circ}\right]$. The key step in this cascade reaction is the aldol reaction between dihydroxyacetone phosphate (DHAP) and an aldehyde catalysed by DHAP-dependent aldolases. A highly stereoselective intramolecular cyclization (Henry reaction) take place during the aldolase catalysed condensation and is followed by a phytase-catalysed phosphate 
hydrolysis step, obtaining enantiomerically pure compounds with four new stereocenters for each aldehyde tested.

\subsection{Tandem reactions employing dihydroxyacetone (DHA)-dependent aldolases.}

Fructose-6-phosphate aldolase (FSA) was described for the first time by Schürmann and Sprenger [45]. This enzyme is a class I aldolase and catalyze the reversible aldol addition of its natural donor, dihydroxyacetone (DHA), on an aldehyde producing 3S,4R diols. Besides, FSA has attracted considerable attention on account of its unique utilization not only of DHA, but also of glycolaldehyde (GA), hydroxyacetone (HA) and hydroxybutanone (HB) as donor compounds. Garrabou et al. [46*] have reported that FSA is able to catalyze direct stereoselective self-aldol addition of GA furnishing D-(-)-threose. Taken advantage of the flexibility shown by FSA with respect to the donor substrate, the same authors described the synthesis of 1-deoxy-D-ido-hept-2-ulose in a multi-substrate cascade reaction of two consecutive aldol additions FSA-catalysed with two different donors. Recently Sánchez-Moreno et al. [47] have designed two straightforward strategies for the synthesis of several sugars monophosphates. These strategies are based on the use of FSA with the four known donors as the key step of a three or four enzyme-catalysed cascade reaction. In both approaches the FSA acceptor substrate, D-glyceraldehyde-3-phosphate (D-G3P), is obtained by isomerization of DHAP using a triosephosphate isomerase. In the first method DHAP was achieved by enzymatic phosphorilation of DHA and the reaction system was completed with a pyruvate kinase for cofactor regeneration. In the second methodology D-G3P was generated from the split of D-fructose-1,6-bisphosphate (FBP) catalysed by a FBP-aldolase (Figure 3C).

\subsection{Tandem reactions employing threonine-dependent aldolases.}

Threonine aldolases catalyze the reversible cleavage of L-threonine to glycine and acetaldehyde and require pyridoxal 5'-phosphate (PLP) as cofactor. These aldolases have been extensively used for the preparation of $\beta$-hydroxy- $\alpha$-amino acids that are medicines by themselves or can be used as chiral building blocks for the production of drugs [48]. Recently, the Griengl's group has developed bienzymatic one-pot synthesis of $\beta$-amino alcohols with high enantiomeric excess by means of a combination of a threonine aldolase-catalysed aldol reaction and a subsequent decarboxylation step tyrosine decarboxylase-catalysed (Figure 3D). In this way, the equilibrium of the aldol reaction is shifted to the side of the aldol product [49].

\subsection{Tandem reactions employing acetaldehyde-dependent aldolases.}

An acetaldehyde-dependent aldolase from Arthrobacter simplex AKU 626 that catalyzes the aldol condensation of acetaldehyde and $\alpha$-ketobutyrate has been used in combination with an aminotransferase for the two-step enzymatic synthesis of 4-hydroxy-L-isoleucine (HIL). In the 
$\mathrm{A}$

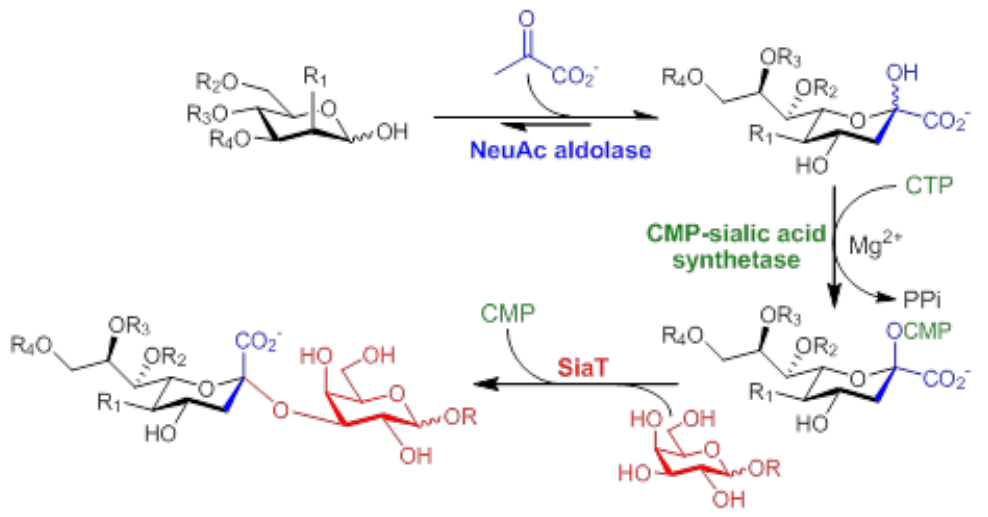

B

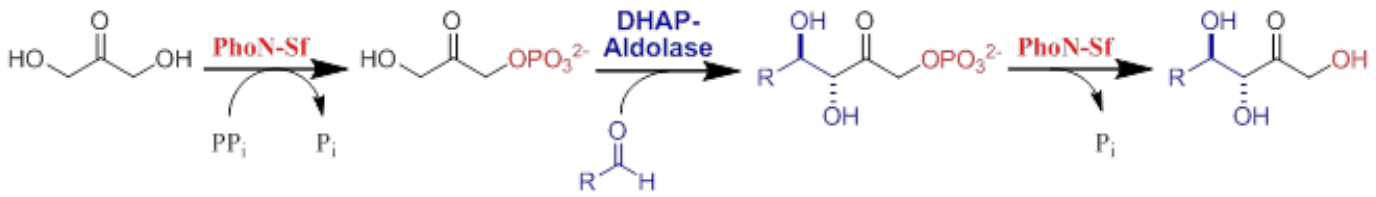

C

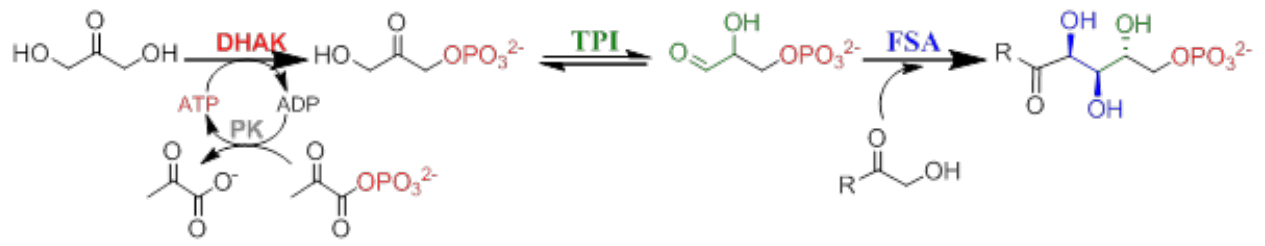

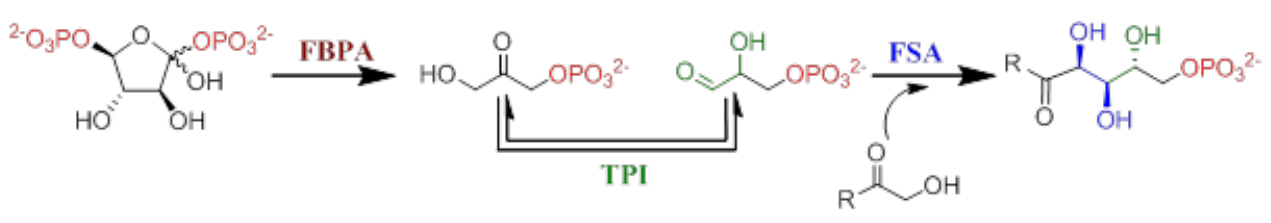

$\mathrm{D}$

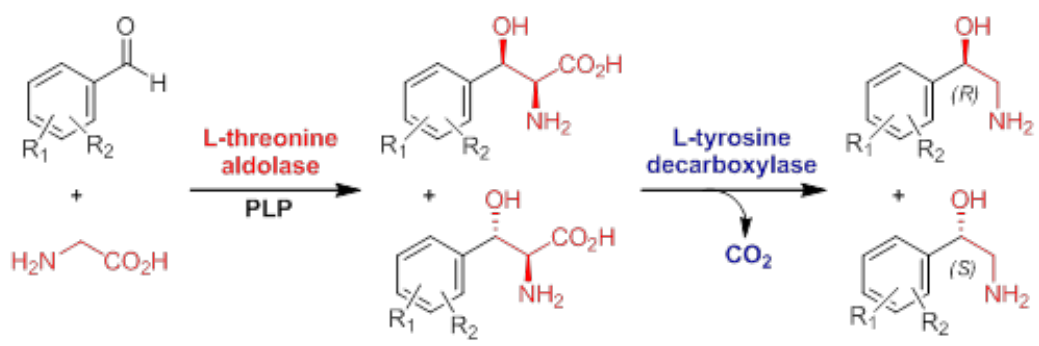

E

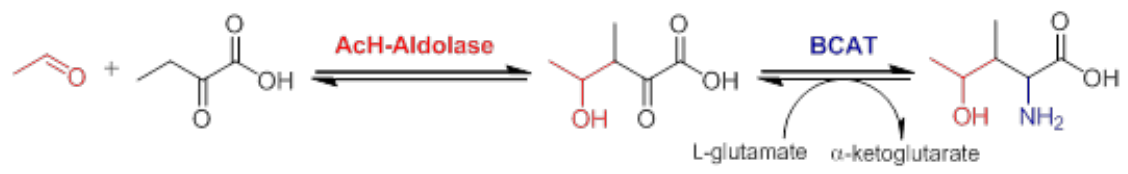

Figure 3. C-C bond formation using aldolases. A. One-pot three-enzyme approach for the synthesis of sialosides using a pyruvate-dependent aldolase. B. Multi-enzyme system to obtain unphosphorilated aldols by DHAP-dependent aldolases using DHA as initial donor. C. Synthesis of monophosphorilated sugars employing a DHA-dependent-aldolase. D. Bienzymatic synthesis of aromatic $\beta$-amino alcohols threonine-dependent aldolase-catalysed. E. Two-step enzymatic synthesis of 4-hydroxy-L-isoleucine utilizing an acetaldehyde-dependent aldolase (AcH-Aldolase). 
first step, 4-hydroxy-3-methyl-2-keto-pentanoate aldolase (HPAL) catalysed the aldol reaction between acetaldehyde and $\alpha$-ketobutyrate. In the second step, amination of 4-hydroxy-3-methyl2-keto-pentanoate (HMKP) by the branched-chain amino acid aminotransferase leads to synthesis of HIL [50] (Figure 3E).

\section{Multi-enzyme synthesis of nucleosides, nucleotides and derivatives.}

Natural nucleosides, nucleotides and their modified derivatives, play a variety of roles in biochemistry, biology, and medicine. They are commonly used for the synthesis of antiviral and antitumor agents as well as for the synthesis of oligonucleotides and glycoconjugates. Multiple enzymatic processes have been developed to produce various sugar-nucleotides from cheap and easily available substrates. Here we report just a few examples:

A recent approach for the synthesis of nucleotide analogues has been reported by Scism and Bachmann [51]. They have developed a covalently linked enzyme aggregate (CLEA). In this pathway ribokinase phosphorylates D-ribose obtaining D-ribose-5-phosphate, which is subsequently pyrophosphorylated by phosphoribosyl pyrophosphate synthetase generating phosphorybosil pyrophosphate (PRPP). Then, the transferase 8B3PRT catalyzes the addition of purine nucleobases to the activated sugar. ATP is regenereted by including adenylate kinase and pyruvate kinase, which uses phosphoenol pyruvate (PEP) as a source of activated phosphate. This method can potentially provide a great variety of nucleotide analogues.

Regeneration of the sugar nucleotide has been carried out to improve enzymatic methods involving glycosyltransferases. Uridine diphosphoglucose (UDP-Glc) is the most commonly encountered sugar nucleotide in biological systems. Starting from raw materials Dong et al. [52] designed a new multi-enzyme system for in vitro synthesis of this compound. Maltodextrin is phosphorylated to glucose monophosphate by maltodextrin phosphorylase. The latter is then converted to UDP-Glc by glucose-1-phosphate thymidylytransferase, generating pyrophosphate (PPi) as a byproduct which is further hydrolyzed to phosphoric acid (Pi) by an inorganic pyrophosphatase. The last step serves to drive the reaction in the UDP-Glc direction and, also, to regenerate one starting product for the maltodextrin phosphorylase reaction. Enzymes were immobilized into amino-functionalized magnetic nanoparticles to facilitate the isolation of the biocatalyst from the reaction media and to improve their stability and reusability.

Specific isotope labelling has many applications in biochemical and biophysical studies of nucleic acid structure and function. Williamson and co-workers [53 ${ }^{\bullet \bullet}$ ] used a total of 28 enzymes in a single-pot reaction for the synthesis of isotopically labelled nucleotides. This methodology couples de novo purine biosynthesis with phosphoribosyl-pyrophosphate synthesis to achieve a total enzymatic synthesis of the purine nucleotides. The driving force of the 
designed biosynthetic pathway were the regeneration systems for $\mathrm{NTP}, \mathrm{NAD}(\mathrm{P}) \mathrm{H}$ and the newly implemented recycling schemes for folate, fumarate, and glutamine cofactor pools.

\section{Multi-ezyme systems for the synthesis of oligosacharides and glycoconjugates}

Oligosaccharides and glyconjugates have numerous biological roles in physiological responses like cell recognition, signalling, infection, adhesion, inflammation, and normal and abnormal cellular developments. Thus, is important to access to defined oligosaccharides to determine the exact biological roles and to exploit their potential use for medical-therapeutical purposes. Access to these molecules has been complicated by a lack of general methods for the routine preparation of this important class of compounds. However, enzyme-based methodologies have greatly simplified synthetic strategies: glycosyltransferases (GTs), Glycoside hydrolases (GHs) and, recently, glycosynthases (GSs) have become valuable catalysts for constructing stereo- and regiospecific glycosidic linkages in oligosaccharide structures [54]. Moreover, enzymes such as sulfotransferases, proteases, lipases, and aldolases can be used as "tailoring enzymes" for the functionalization of the oligosaccharide scaffold.

Glycosyltransferases catalyze the formation of glycosidic linkages by the transfer of a saccharide from a donor substrate to an acceptor substrate. These enzymes use either sugar nucleotides or glycosyl phosphates as their activated sugar donors. Acceptors can be other saccharides, proteins, lipids, nucleic acids, natural products and unnatural products. Although they have high stereospecificity and regiospecificity GTs suffer from two major drawbacks: first, activated donors of the most common monosaccharides are commercially available but are expensive and, furthermore, the nucleoside diphosphates generated during the reaction are potent glycosyltransferase inhibitors. Hence, several recycling systems have been developed. The second issue is the availability of glycosyltransferases because every glycosidic linkage needs its own enzyme. In spite of these disadvantages, GTs have been widely used in the synthesis of oligosaccharides and glyconjugates [55].

The use of bacterial GTs to synthesize mammalian structures is exemplified in a three-step synthesis to produce Forssman antigen pentasaccharide from lactose-pNP using novel $\alpha 1,4$ GalT and $\beta 1,3$-GalNAcT from Campylobacteri jejuni and a novel $\alpha 1,3-G a l N A c T$ from Pasteurella multocida. UDP-GalNAc epimerase was employed in this synthesis to allow the use of less costly UDP-GlcNAc donor [56]. Even more complex oligosaccharides can be obtained: libraries of heparin sulfates from heptasaccharides to dodecasaccharides were produced by the use of two glycosyltransferases for the linear backbone followed by selective enzymatic $O$ sulfation and $N$-sulfation and C-5-epimerization [57 ${ }^{\bullet \bullet}$. The production of 24 different blood 
group and tumor associated antigens has been achieved using and six different GTs enzymes, starting GlcNAc chemically synthesized. The monosaccharide was elaborated to LacNAc disaccharide with a recombinant Neisseria meningitidis $\beta 1,4-G a l T$ fused with a UDPGal epimerase enzyme, which allows the use of less expensive UDP-Glc as donor. The product was converted to poly- $N$-acetyllactosamine repeating units by the action of $\beta 1,3-G l c N A c T$ and $\beta 1,4-$ GalT and then all the LacNAc-terminating compounds were converted to either $\alpha 2,3$-sialylated, a2,6-sialylated, $\alpha 1,2$-fucosylated or $\alpha 1,3$-fucosylated with fucosyltransferases and sialyltransferases [58]. In an elegant way to obtain glycopeptides, Huang et al. [59] have successfully demonstrated a multi-enzyme one-pot glycosylation strategy for the synthesis of sLe ${ }^{\mathrm{x}}$-containing truncated PSGL-1 glycopeptides, with or without sulfation. In the first enzymatic step glycopeptide was treated with $\beta$-1,4-galactosyltransferase and $\alpha-2,3-$ sialyltransferase in the presence of donor substrates UDP-Gal and CMP-NeuAc. Subsequently, the resultant product was incubated with $\alpha-1,3$ - fucosyltransferase and GDP-Fuculose. Alkaline phosphatase was added to all the reactions to reduce the product inhibition caused by the pyrophosphate group.

Recently, Serna et al. [60] have reported an effective chemoenzymatic strategy for the construction of glycans microarrays. Firstly, a variety of $\mathrm{N}$-glycan core structures have been chemically synthesized and covalently immobilized on a glass surface. Then, glycans were enzymatically decorated in reactions carried out in nanodroplets using three different glycosyltransferases. By using this methodology a great diversity of synthetic oligosaccharides were prepared.

Glycosidases are the second large class of carbohydrate processing enzymes, catalyzing the hydrolysis of glycosidic linkages in vivo. Nevertheless, under controlled conditions, glycosidases have been used in vitro to synthesize glycosidic bonds. In comparison with glycosyltransferase-catalysed reaction they have several advantages, including a broad substrate range, the use of readily available donor substrates and the fact that they are generally more robust and accessible. Despite these advantages the use of glycosidases in synthesis is subject to three major limitations: the low regiospecificity that may result in the formation of multiple products, the low transglycosylation yield and the product hydrolysis. A strategy to avoid the hydrolysis of the product formed is its entrapment by glycosylation with a glycosyltransferase, producing a saccharide that is no longer hydrolyzed by the glycosidase. This system was optimized by Hoh et al. [61] using a genetic algorithm to generate the core 2-benzyl trisaccharide using a $\beta$-galactosidase and a $\beta$-GlcNAc-transferase as a multienzyme system with $90 \%$ yield. 
Glycosynthases are artificial mutant glycosidases hydrolytically inactive but that can readily form glycosidic linkages transfering an activated sugar donor, such as a glycosyl fluoride, to a suitable acceptor substrate [62]. The utilization of these mutants results in high yields transglycosilation and address the lack of glycosyltransferases availability. In a recent example Spadiut et al. [63] have developed a new "xyloglucan glycosynthase", which catalyzes the synthesis of high molar mass polysaccharides, with complex side-chain structures, from suitable glycosyl fluoride donor substrates. The combination of this new glycosynthase with an Arabidopsis thaliana a1,2-fucosyltransferase leads to a toolkit for the controlled enzymatic synthesis of xyloglucans.

\section{Energy production}

\subsection{Tandem reactions for the production of biofuels.}

Production of renewable energy, replacing the use of non-renewable fossil fuels, represents a major challenge in global efforts to afford a sustainable development of humankind. Biomass is the most abundant renewable bioresource and can be used for the production of large amounts of biofuels and renewable materials at affordable costs. Starting from biomass sugars, numerous biocatalytic approaches have been proposed and investigated for the production of biofuels. High-yield ethanol production was produced by a reconstituted yeast glycolytic enzyme system containing ATPase, which facilitates complete conversion of glucose to ethanol. Alternatively, substitution of arsenate for inorganic phosphate can constrain ATP synthesis and prevent ATP accumulation in this reaction. In the same way, butanol can also be produced mimicking butanol fermentation pathway. Both approaches are achieved by using cell-free synthetic pathway biotransformation [64].

\subsection{Multi-enzyme production of hydrogen.}

Hydrogen is a promising future clean energy carrier with high-energy utilization efficiency. Hydrogen production from renewable energy sources, like biomass sugars, is highly demanded and a shortcut for producing low-cost hydrogen without net carbon emissions. The feasibility of producing high-yield hydrogen from starch has been demonstrated by Zhang and coworkers [65] using a 13 enzymes cocktail. However, this technology suffers from low hydrogen yield due to incomplete reaction. To overcome the task of producing high-yield hydrogen, a new multi-enzyme system was designed by this group. This system contains five sub-modules: (i) conversion of cellobiose into glucose-1-phosphate (G-1P) catalysed by cellobiose phosphorylase, (ii) generation of glucose-6-phosphate (G-6P) from G-1P catalysed by phosphoglucomutase, (iii) production of NADPH catalysed by two dehydrogenases of the oxidative phase of the pentose phosphate pathway (PPP), (iv) regeneration of G-6P from 
ribulose-5-phosphate catalysed by the eight enzymes of the nonoxidative phase of PPP, and (v) generation of hydrogen from NADPH catalysed by hydrogenase. This multi-enzyme system generates nearly 12 moles of hydrogen per mole of glucose unit of starch or cellulosic materials and represent about a 10-fold enhancement in hydrogen production rate with respect to the first multi-enzyme system [66 ${ }^{\bullet \bullet}$ ] (Figure 4).

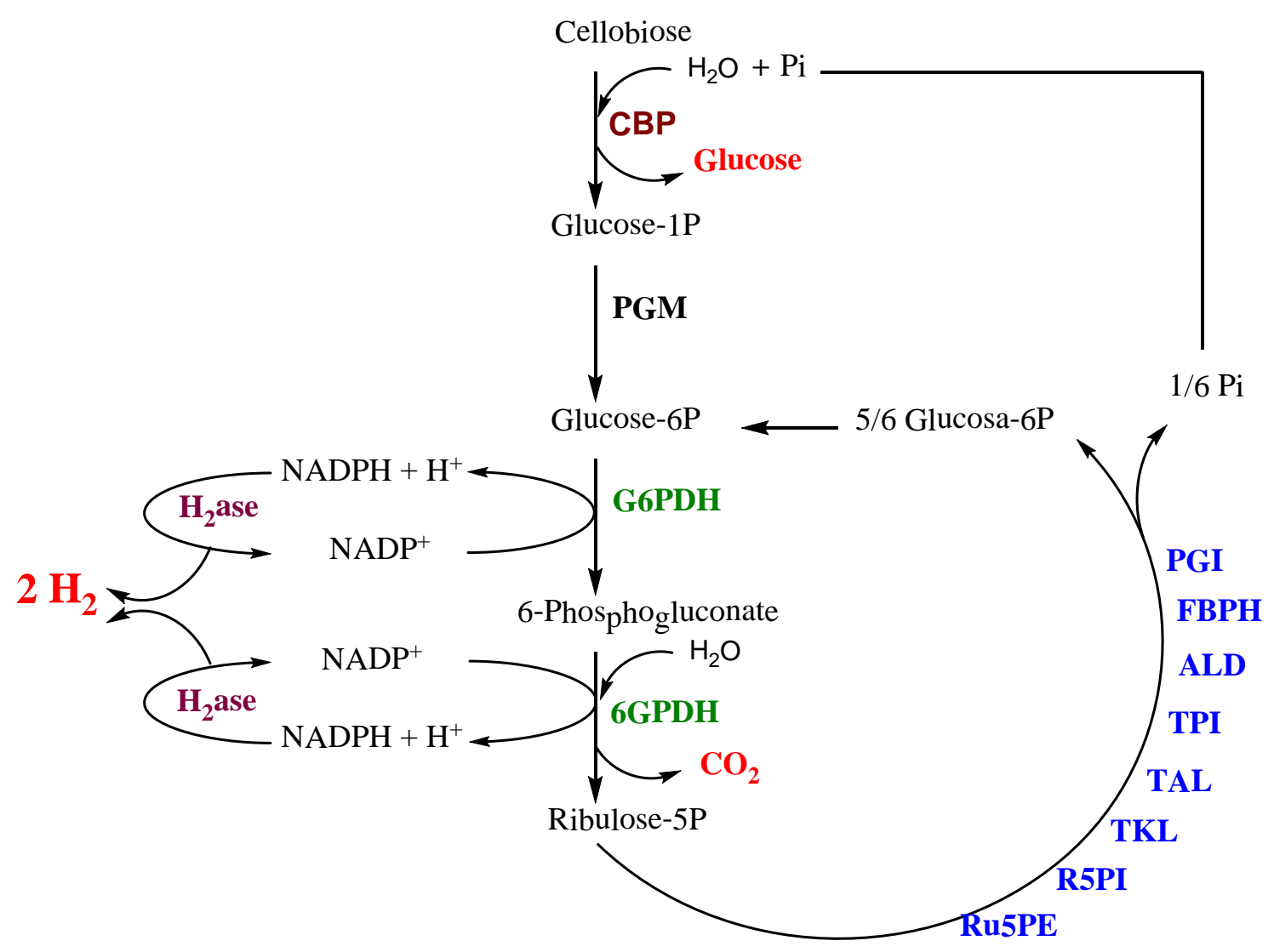

Figure 4. Multi-enzyme production of hydrogen starting from cellobiose and water. The enzymes used were: 1 ) $\mathrm{CBP}=$ cellobiose phosphorylase; 2) $\mathrm{GM}=$ phosphoglucomutase; 3$) \mathrm{G} 6 \mathrm{PDH}=$ glucose-6phosphate dehydrogenase; 4) 6PGDH = 6-phosphogluconate dehydrogenase; 5) R5PI = phosphoribose isomerase; 6) Ru5PE = ribulose 5-phosphate epimerase; 7) TKL = transketolase; 8) TAL = transaldolase; 9) $\mathrm{TPI}=$ triose phosphate isomerase; 10) ALD = aldolase; 11) FBPH= fructose-1,6-bisphosphatase; 12) PGI = phosphoglucose isomerase; and 13) $\mathrm{H}_{2}$ ase = hydrogenase. This pathway is divided in five submodules: (i) conversion of cellobiose into glucose-1P (dark red-colored); (ii) generation of glucose-6phosphate (black-colored); (iii) production of NADPH (green-colored); (iv) regeneration of glucose-6P (blue-colored) and (v) generation of hydrogen from NADPH catalysed by hydrogenase (violet-colored). Products obtained are colored in red.

\subsection{Multi-enzyme biofuel cells.}

Enzymatic biofuel cells are a type of fuel cell where chemical energy is converted to electrical energy by employing enzymes as the electrocatalysts. These biofuel cells offer significant 
potential advantages to traditional fuel cells: (i) the nanoscale of enzymes greatly improves the volumetric catalytic activity in comparison to microorganisms, (ii) impurities in the fuel typically do not interfere with the enzymes allowing a wide variety of fuels to be utilized without the need for expensive and time consuming purification, (iii) a simultaneous use of a bio-cathode and a bio-anode can be implemented due to the selectivity of the enzymes. However, these systems have some limitations including incomplete oxidation of fuels, short lifetimes, and reduced performance due to slow direct electron transfer kinetics or problems associated with the stability or thermodynamics of redox mediators [67]. One possibility to overcome those drawbacks is the use of immobilized multi-enzyme systems, enhancing the stability of the enzymes, favoring the completely oxidation of the fuel and increasing the energetic reaction yield.

The first biofuel-based multistep oxidation of alcohols was reported by Palmore et al. [68] showing that a three-enzyme cascade consisting on alcohol dehydrogenase, formaldehyde dehydrogenase and formate dehydrogenase could be used for complete oxidation of methanol to carbon dioxide. Besides, a fourth enzyme, diaphorase, was added to allow the regeneration of $\mathrm{NAD}+$ from NADH by reducing the mediator, benzyl viologen. In another approach, the complete oxidation of glycerol was achieved by Arechederra and Minteer [69] through the sequential action of alcohol dehydrogenase, aldehyde dehydrogenase and oxalate oxidase. Recently, Xu and Minteer [70 ${ }^{\circ}$ ] have design an artificial metabolic pathway but avoiding almost all the enzyme-catalysed steps that do not result in electricity generation. Thus, the six enzyme cascade - consists on a pyrroloquinoline quinine (PQQ)-dependent glucose dehydrogenase, a PQQ-dependent 2-gluconolactone dehydrogenase, an aldolase, an alcohol dehydrogenase, an aldehyde dehydrogenase and a oxalate oxidase-, allows the complete oxidation of glucose.

\section{Conclusions}

Multi-enzyme in vitro cascade reactions have risen as an important area in the field of biocatalysis. This is due to the extraordinary level of control and surprising diversity of approaches available for building biosynthetic systems without constraints that limit cellular engineering. In vitro reactions with isolated biocatalysts have been successfully used for the synthesis of complex molecules that frequently are not readily accessible by organic chemical synthesis. Novel products can be generated by different enzymes combinations, which are often from different organisms and by using natural and unnatural starting substrates expanding the range of biosynthetic compounds obtained. In the future the coupling of enzymes in multienzyme cascade processes will become much more common due to: (i) the ability to mimic the new metabolic pathways found in living organisms, (ii) the increased number of new enzymes that can be obtaining, characterized and potentially used as biocatalysts in synthesis, (iii) the 
improvement of the overexpression, isolation, stabilization and compartmentalization techniques, (iv) the use of protein engineering techniques to extend its operating window and (v) the optimization of the reaction conditions. All these approaches will result in biocatalytic cascade processes of unprecedented complexity, increasing reaction rates and efficiency and being an important step for a "greener" chemical future.

\section{BIBLIOGRAFÍA}

1. Sáchez-Moreno I, Oroz-Guinea I, Iturrate L, García-Junceda E: 7.20 Multi-enzyme reactions. In Comprehensive Chirality, Edited by Carreira EM, Yamamoto H. vol. 7 Synthetic Methods VI - Enzymatic and Semi-Enzymatic Edited by Turner N Elsevier.; 2012:430-453. (Amsterdam).

- This chapter summarizes recent strategies and methods developed in multienzyme synthesis of chiral compounds.

2. López-Gallego F, Schmidt-Dannert C: Multi-enzymatic synthesis. Curr Opin Chem Biol 2010, 14:174-183.

- Excellent review that illustrates the benefits, disadvantages and challenges for in vivo and in vitro multi-enzyme synthesis approaches.

3. Truppo MD: Cofactor Recycling for Enzyme Catalyzed Processes. In Comprehensive Chirality, Edited by Carreira EM, Yamamoto H. vol. 7 Synthetic Methods VI - Enzymatic and Semi-Enzymatic Edited by Turner N Elsevier; 2012:4670. (Amsterdam).

4. Sheldon RA: Enzyme catalyzed cascade reactions. In Multi-Step Enzyme Catalysis: Biotransformations and Chemoenzymatic Synthesis. Edited by García-Junceda E, Wiley-VCH, 2008: 109-135 (Weinheim).

5. Wada M, Yoshizumi A, Noda Y, Kataoka M, Shimizu S, Takagi H, Nakamori S: Production of a Doubly Chiral Compound, (4R,6R)-4-Hydroxy-2,2,6Trimethylcyclohexanone, by Two-Step Enzymatic Asymmetric Reduction. Appl Environ Microbiol 2003, 69:933-937.

6. Ma SK, Gruber J, Davis C, Newman L, Gray D, Wang A, Grate J, Huisman GW, Sheldon RA: A green-by-design biocatalytic process for atorvastatin intermediate. Green Chemistry 2010, 12:81-86. 
7. Kalaitzakis D, Smonou I: A two-step, one-pot enzymatic synthesis of 2-substituted 1,3-diols. J Org Chem 2010, 75:8658-8661.

8. Voss CV, Gruber CC, Faber K, Knaus T, Maceroux P, Kroutil W: Orchestration of Concurrent Oxidation and Reduction Cycles for Stereoinversion and Deracemisation of sec-alcohols. J Am Chem Soc 2008, 130:13969-13972.

-• An interesting deracemization and stereoinversion of sec-alcohols are achieved by combination of four oxidoreductases in a concurrent oxidation/reduction processes involving opposite cofactor recycling systems.

9. Bisogno FR, Rioz-Martínez A, Rodríguez C, Lavandera I, de Gonzalo G, Torres Pazmiño DE, Fraaije MW, Gotor V: Oxidoreductases Working Together: Concurrent Obtaining of Valuable Derivatives by Employing the PIKAT Method. ChemCatChem 2010, 2:946-949.

10. Rioz-Martínez A, Bisogno FR, Rodríguez C, de Gonzalo G, Lavandera I, Torres Pazmiño DE, Fraaije MW, Gotor V: Biocatalysed concurrent production of enantioenriched compounds through parallel interconnected kinetic asymmetric transformations. Org Biomol Chem 2010, 8:1431-1437.

- In this work, the authors describe the concurrent production of enantioenriched ketones, sulfoxides and sec-alcohols in a strict parallel way, using the parallel interconnected kinectic asymmetric transformations (PIKAT) methodology.

11. Monti D, Ferrandi EE, Zanellato I, Hua L, Polentini F, Carrea G, Riva S: One-Pot Multienzymatic Synthesis of 12-Ketoursodeoxycholic Acid: Subtle Cofactor Specificities Rule the Reaction Equilibria of Five Biocatalysts Working in a Row. Adv Synth Catal 2009, 351:1303-1311.

12. Carlson JC, Li S, Gunatilleke SS, Anzai Y, Burr DA, Podust LM, Sherman DH: Tirandamycin biosynthesis is mediated by co-dependent oxidative enzymes. Nat Chem 2012, 3:628-633.

13. Clapés P, Garrabou X: Current Trends in Asymmetric Synthesis with Aldolases. Adv Synth Catal 2011, 353:2263-2283.

- A comprehensive review covering recent progress in C-C bond formation mediated by aldolases and the perspectives in this field. 
14. Mateo C, Chmura A, Rustler S, van Rantwijk F, Stolzb A, Sheldon RA: Synthesis of enantiomerically pure (S)-mandelic acid using an oxynitrilase-nitrilase bienzymatic cascade: a nitrilase surprisingly shows nitrile hydratase activity. Tetrahedron: Asymmetry 2006, 17:320-323.

15. Xu Y, Jia X, Pankeb S, Li Z: Asymmetric dihydroxylation of aryl olefins by sequential enantioselective epoxidation and regioselective hydrolysis with tandem biocatalysts. Chem Commun 2009, 1481-1483.

16. di Gennaro P, Bernasconi S, Orsini F, Corretto E, Sello G: Multienzymatic preparation of 3-[(1R)-1-hydroxyethyl]benzoic acid. Tetrahedron: Asymmetry 2010, 21:1885-1889.

17. Ankati H, Zhu D, Yang Y, Biehl ER, Hua L: Asymmetric Synthesis of Both Antipodes of $\beta$-Hydroxy Nitriles and $\beta$-Hydroxy Carboxylic Acids via Enzymatic Reduction or Sequential Reduction/Hydrolysis. J Org Chem 2009, 74:1658-1662.

- Optically pure $(R)$ - and (S)- $\beta$-hydroxy carboxylic acids were obtained in high yields in a two-step/one-pot process via enzymatic reduction and hydrolysis of a $\beta$ ketonitrile. The use of isolated enzymes eliminates the competing $\alpha$-ethylation that is present when whole cell biocatalysts were used.

18. van Pelt S, van Rantwijk F, Sheldon RA: Synthesis of Aliphatic (S)-aHydroxycarboxylic Amides using a One-Pot Bienzymatic Cascade of Immobilised Oxynitrilase and Nitrile Hydratase. Adv Synth Catal 2009, 351:397404.

19. Höhne M, Bornscheuer UT: Biocatalytic Routes to Optically Active Amines. ChemCatChem 2009, 1:42-51.

20. Koszelewski D, Tauber K, Faber K, Kroutil W: Enzymatic racemization of amines catalyzed by enantiocomplementary $\omega$-transaminases. Trends Biotechnol 2010, 28:324-332.

21. Resch V, Fabian WMF, Kroutil W: Deracemisation of Mandelic Acid to Optically Pure Non-Natural-Phenylglycine via a Redox-Neutral Biocatalytic Cascade. Adv Synth Catal 2010, 352:993-997. 
- The authors have developed a linear redox-neutral cascade in which both, the substrate and the cofactor recycling are provided via enzymatic oxidation of an $\alpha$ hydroxy acid.

22. Nakajima N, Esaki N, Soda K: Enzymatic conversion of racemic methionine to the L-enantiomer. Chem Commun 1990, 947-948.

23. Findrik Z, Vasić-Rački D: Biotransformation of D-methionine into L-methionine in the cascade of four enzymes. Biotechnol Bioeng 2007, 98:956-967.

24. Koszelewski D, Clay D, Rozzel JD, Kroutil W: Deracemisation of $\boldsymbol{\alpha}$-Chiral Primary Amines by a One-Pot, Two-Step Cascade Reaction Catalysed by $\omega$ Transaminases. Eur J Org Chem 2009, 2289-2292.

25. Truppo MD, Turner NJ, Rozzell JD: Efficient kinetic resolution of racemic amines using a transaminase in combination with an amino acid oxidase. Chem Commun 2009, 2127-2129.

- The authors have developed a new method for kinetic resolution of amines using amino transferases requiring only catalytic quantities of the amine acceptor pyruvate that is continuously recycled.

26. Koszelewski D, Grischek B, Glueck SM, Kroutil W, Faber K: Enzymatic Racemization of Amines Catalyzed by Enantiocomplementary $\omega$ Transaminases. Chem Eur J 2011, 17: 378-383.

27. Cho BK, Cho HJ, Park SH, Yun H, Kim, BG: Simultaneous Synthesis of Enantiomerically Pure (S)-Amino Acids and (R)-Amines Using Coupled Transaminase Reactions. Biotechnol Bioeng 2003, 81:783-789.

28. Hong EY, Cha M, Yunb H, Kim BG: Asymmetric synthesis of L-tert-leucine and L3-hydroxyadamantylglycine using branched chain aminotransferase. $J$ Mol Catal B: Enzym 2010, 66:228-236.

29. Bae HS, Lee SG, Hong SP, Kwak MS, Esaki N, Soda K, Sung MH: Production of Damino acids from $\alpha$-keto acids and ammonia by coupling of four enzyme reactions. J Mol Catal B: Enzym 1999, 6:241-247. 
30. Gu X, Xian M, Roy-Faure S, Bolte J, Aitken DJ, Gefflaut T: Synthesis of the constrained glutamate analogues (2S,1'R,2'R)- and (2S,1'S,2'S)-2-(2'carboxycyclobutyl)glycines L-CBG-II and L-CBG-I by enzymatic transamination. Tetrahedron Lett 2006, 47:193-196.

31. Caligiuri A, D’Arrigo P, Gefflaut T, Molla G, Pollegioni L, Rosini E, Rossi C, Servi S: Multistep enzyme catalysed deracemisation of 2-naphthyl alanine. Biocatal. Biotransform 2006, 24:409-413.

32. Turner NJ: Ammonia lyases and aminomutases as biocatalysts for the synthesis of $\boldsymbol{\alpha}$-amino and $\boldsymbol{\beta}$-amino acids. Curr Opin Chem Biol 2011, 15:234-240.

33. Paizs C, Katona A, Rétey: Chemoenzymatic One-Pot Synthesis of Enantiopure LArylalanines from Arylaldehydes. Eur J Org Chem 2006, 1113-1116.

34. Cox BM, Bilsborrow JB, Walker KD: Enhanced conversion of racemic alphaarylalanines to $(R)$-beta-arylalanines by coupled racemase/aminomutase catalysis. J Org Chem 2009, 74:6953-6959.

35. Wu B, Szymanski W, de Wildeman S, Poelarends GJ, Feringa BL, Janssen DB: Efficient tandem biocatalytic process for the kinetic resolution of aromatic $\beta$ amino acids. Adv Synth Catal 2010, 352:1409-1412.

36. Wang TH, Chen YY, Pan HH, Wang FP, Cheng CH, Lee WC: Production of $\mathrm{N}$ acetyl-D-neuroaminic acid using two sequential enzymes overexpressed as double-tagged fusion proteins. BMC Biotechnol 2009, 9:63.

37. Yu H, Cheng J, Ding L, Khedri Z, Chen Y, Chin S, Lau K, Tiwari VK, Chen X: Chemoenzymatic synthesis of GD3 oligosaccharides and other disialyl glycans containing natural and non-natural sialic acids. $J$ Am Chem Soc 2009, 131:1846718477.

38. Cao HZ, Li YH, Lau K, Muthana S, Yu H, Cheng JS, Chokhawala HA, Sugiarto G, Zhang L, Chen X: Sialidase substrate specificity studies using chemoenzymatically synthesized sialosides containing C5-modified sialic acids. Org Biomol Chem 2009, 7:5137-5145. 
39. Sánchez-Moreno I, García-García JF, Bastida A, García-Junceda E: Multienzyme system for dihydroxyacetone phosphate-dependent aldolase catalyzed $\mathrm{C}-\mathrm{C}$ bond formation from dihydroxyacetone. Chem. Commun 2004, 1634-1635.

40. Sánchez-Moreno I, Iturrate L, Martín-Hoyos R, Jimeno ML, Mena M, Bastida A, García-Junceda E: From Kinase to Cyclase: An Unusual Example of Catalytic Promiscuity Modulated by Metal Switching. ChemBioChem, 2009, 10, 225-229.

- The natural catalytic promiscuity of the C. freundii DHAK is described. This enzyme is able to catalyse the transfer of the $\gamma$-phosphate of the ATP to DHA and the cyclization of the FAD to yield riboflavin 4',5'-cyclic phosphate (4',5'-cFMN). The mechanism for switching between cyclase and kinase activities is the change of the divalent cation that forms the complex with the phosphorylated substrate. This work shown that catalytic promiscuity provide a mechanism for enzyme evolution, with the old function maintained, the new one evolving, and without gene duplication necessary

41. Sánchez-Moreno I, Iturrate L, Doyagüez EG, Martínez JA, Fernández-Mayoralas A, García-Junceda E: Activated $\boldsymbol{\alpha , \beta}$-Unsaturated Aldehydes as Substrate of Dihydroxyacetone Phosphate (DHAP)-Dependent Aldolases in the Context of a Multienzyme System. Adv Synth Catal 2009, 351:2967-2975

42. Oroz-Guinea I, Sánchez-Moreno I, García-Junceda E: Aldolase based multi-enzyme system for carbon-carbon bond formation. In Practical Methods in Biocatalysis and Biotransformations. Edited by Whittall J, Sutton P, John Wiley \& sons 2012: 323-331.

- Design of a versatile multi-enzyme system for one-pot C-C bond formation catalysed by DHAP-dependent aldolases and based on the use of the recombinant ATP-dependent DHAK from C. freundii CECT 4626 for the in situ phosphorylation of DHA.

43. van Herk T, Hartog AF, Schoemaker HE, Wever R: Simple enzymatic in situ generation of dihydroxyacetone phosphate and its use in a cascade reaction for the production of carbohydrates: increased efficiency by phosphate cycling. $J$ Org Chem 2006, 71:6244-6247.

44. El Blidi L, Assaf Z, Camps-Bres F, Veschambre H, Théry V, Bolte J, Lemaire M: Fructose-1,6-Bisphosphate Aldolase-Mediated Synthesis of Aminocyclitols 
(Analogues of Valiolamine) and their Evaluation as Glycosidase Inhibitors. ChemCatChem 2009, 1:463-471.

- The work presented in this paper is an efficient synthesis of new nitro- and aminociclitols opening a new route for the synthesis of valiolamine analogues. Enantiomerically pure compounds were obtained with four new stereocenters.

45. Schürmann M, Sprenger GA: Fructose-6-phosphate aldolase is a novel class $\mathbf{I}$ aldolase from Escherichia coli and is related to a novel group of bacterial transaldolases.. J Biol Chem 2001, 276:11055-11061.

46. Garrabou X, Castillo JA, Guérard-Hélaine C, Parella T, Joglar J, Lemaire M, Clapés P: Asymmetric Self- and Cross-Aldol Reactions of Glycolaldehyde Catalyzed by D-Fructose-6-phosphate Aldolase. Angew Chem Int Ed. 2009, 48:5521-5525.

- In this work a new general activity of FSA, unprecedented for ketose-dependent aldolases, is described. Base on this newly discovered activity, the authors develop a new two-step cascade strategy for the direct syn cross-aldol addition of glycolaldehyde to aldehydes.

47. Sánchez-Moreno I, Hélaine V, Poupard N, Charmantray F, Légeret B, Hecquet L, García-Junceda E, Wohlgemuth R, Guérard-Hélaine C, Lemaire M: One-Pot Cascade Reactions using Fructose-6-phosphate Aldolase: Efficient Synthesis of D-Arabinose 5-Phosphate, D-Fructose 6-Phosphate and Analogues. Adv Synth Catal 2012, 354:1725-1730.

48. Dückers N, Baer K, Simon S, Gröger H, Hummel W: Threonine aldolases screening, properties and applications in the synthesis of non-proteinogenic $\beta$ hydroxy-a-amino acids. Appl Microbiol Biotechnol 2010, 88:409-424.

49. Steinreiber J, Schürmann M, Wolberg M, van Assema F, Reisinger C, Fesko K, Mink D. Griengl H: Overcoming thermodynamic and kinetic limitations of aldolasecatalyzed reactions by applying multienzymatic dynamic kinetic asymmetric transformation. Angew Chem Int Ed 2007, 46:1624-1626.

50. Smirnov SV, Samsonova NN, Novikova AE, Matrosov NG, Rushkevich NY, Kodera T, Ogawa J, Yamanaka H, Shimizu S: A novel strategy for enzymatic synthesis of 4-hydroxyisoleucine: identification of an enzyme possessing HMKP (4-hydroxy- 
3-methyl-2-keto-pentanoate) aldolase activity. FEMS Microbiol Lett 2007, 273:7077.

51. Scism RA, Bachmann BO: Five-Component Cascade Synthesis of Nucleotide Analogues in an Engineered Self-Immobilized Enzyme Aggregate. ChemBioChem 2010, 11:67-70.

52. Dong Q, Ouyang LM, Yu HL, Xu JH: Efficient biosynthesis of uridine diphosphate glucose from maltodextrin by multiple enzymes immobilized on magnetic nanoparticles. Carbohydr Res 2010, 345:1622-1626.

53. Schultheisz HL, Szymczyna BR, Scott LG, Williamson JR: Pathway Engineered Enzymatic de Novo Purine Nucleotide Synthesis. ACS Chem Biol 2008, 3:499-511. - This paper describes the one-pot in vitro enzymatic synthesis of isotopically labelled nucleotides involving a complex biosynthetic scheme with 28 enzymes and using as driving force the cofactor regeneration systems.

54. Muthana S, Cao $\mathrm{H}$, Chen $\mathrm{X}$ : Recent progress in chemical and chemoenzymatic synthesis of carbohydrates. Curr Opin Chem Biol 2009, 13:573-581.

55. Palcic MM: Glycosyltransferases as biocatalysts. Curr Opin Chem Biol 2011, 15:226-233.

56. Houliston RS, Bernatchez S, Karwaski MF, Mandrell RE, Jarrell HC, Wakarchuk WW, Gilbert M: Complete chemoenzymatic synthesis of the Forssman antigen using novel glycosyltransferases identified in Campylobacter jejuni and Pasteurella multocida. Glycobiology 2009, 19:153-159.

57. Xu Y, Masuko S, Takieddin M, Xu H, Liu R, Jing J, Mousa SA, Linhardt RJ, Liu J: Chemoenzymatic Synthesis of Homogeneous Ultralow Molecular Weight Heparins. Science 2011, 334:498-501.

-• Glycotransferases and sacharide modifying enzymes have been employed in the production of libraries of heparin sulfates starting from a simple disaccharide in a 10or 12-step chemoenzymatic synthesis

58. Vasiliu D, Razi N, Zhang Y, Jacobsen N, Allin K, Liu X, Hoffmann J, Bohorov O, Blixt O: Large-scale chemoenzymatic synthesis of blood group and tumor- 
associated poly- $N$-acetyllactosamine antigens. Carbohydr Res 2006, 341:14471457.

59. Huang KT, Wu BC, Lin CC, Luo SCC, Chen C, Wong CH, Lin CC: Multi-enzyme one-pot strategy for the synthesis of sialyl Lewis ${ }^{\mathrm{x}}$-containing PSGL-1 glycopeptide. Carbohydr Res 2006, 341:2151-2155.

60. Serna S, Etxebarria J, Ruiz N, Martin-Lomas M, Reichardt N-C: Construction of NGlycan Microarrays by Using Modular Synthesis and On-Chip Nanoscale Enzymatic Glycosylation. Chem Eur J 2010, 16:13163-13175.

61. Hoh C, Dudziak G, Liese A: Optimization of the Enzymatic Synthesis of $\boldsymbol{O}$ Glycan Core 2 Structure by Use of a Genetic Algorithm. Bioorganic Med Chem Lett 2002, 12:1031-1034.

62. Kittl R, Withers SG: New approaches to enzymatic glycoside synthesis through directed evolution. Carbohydr Res 2010, 345:1272-1279.

63. Spadiut O, Ibatullin FM, Peart J, Gullfot F, Martinez-Fleites C, Ruda M, Xu C, Sundqvist G, Davies GJ, Brumer H: Building Custom Polysaccharides in Vitro with an Efficient, Broad-Specificity Xyloglucan Glycosynthase and a Fucosyltransferase. J Am Chem Soc 2011, 133:10892-10900.

64. Zhang Y-HP, Sun J, Zhong JJ: Biofuel production by in vitro synthetic enzymatic pathway biotransformation. Curr Opin Biotechnol 2010, 21:663-669.

65. Zhang Y-HP, Evans BR, Mielenz JR, Hopkins RC, Adams MWW: High-yield hydrogen production from starch and water by a synthetic enzymatic pathway. PLoS One 2007, 2:e456.

66. Ye X, Wang Y, Hopkins RC, Adams MWW, Evans BR, Mielenz JR, Zhang Y-HP: Spontaneous high-yield production of hydrogen from cellulosic materials and water catalyzed by enzyme cocktails. ChemSusChem 2009, 2:149-152.

-• The authors designed a multi-enzyme system for hydrogen production, obtaining nearly 12 moles of hydrogen per mol of glucose of unit of cellulosic materials and highly increasing the production rates. 
67. Lapinsonnière L, Picot M, Barrière F: Enzymatic versus Microbial Bio-Catalyzed Electrodes in Bio-Electrochemical Systems. ChemSusChem 2012, 5:995-1005.

68. Palmore GTR, Bertschy H, Bergens SH, Whitesides GM: A methanol/dioxygen biofuel cell that uses $\mathrm{NAD}^{+}$-dependent dehydrogenases as catalysts: application of an electro-enzymatic method to regenerate nicotinamide adenine dinucleotide at low overpotentials. $J$ Electroanal Chem 1998, 443:155-161.

69. Arechederra RL, Minteer SD: Complete Oxidation of Glycerol in an Enzymatic Biofuel Cell. Fuel Cells 2009, 1:63-69.

70. Xu S, Minteer SD: Enzymatic Biofuel Cell for Oxidation of Glucose to $\mathrm{CO}_{2}$. ACS Catal 2012, 2:91-94.

- The originality of this work resides in the construction of an artificial enzymatic cascade that strictly does not mimic a traditional metabolic pathway, leading just a few little non-oxidoreduction reactions. 